\title{
Effects of stathmin 1 silencing by siRNA on sensitivity of esophageal cancer cells Eca-109 to paclitaxel
}

\author{
H.W. Zhu, D. Jiang, Z.Y. Xie, M.H. Zhou, D.Y. Sun and Y.G. Zhao \\ Department of Gastroenterology, Guangzhou General Hospital of the Guangzhou \\ Military Command of the People's Liberation Army, Guangzhou, China \\ Corresponding author: Y.G. Zhao \\ E-mail: zhaoygang666@yeah.net \\ Genet. Mol. Res. 14 (4): 18695-18702 (2015) \\ Received August 6, 2015 \\ Accepted October 18, 2015 \\ Published December 28, 2015 \\ DOI http://dx.doi.org/10.4238/2015.December.28.18
}

\begin{abstract}
We investigated the effects of stathmin 1 (STMN1) silencing by small interfering (siRNA) on the sensitivity of esophageal cancer cells Eca-109 to paclitaxel. STMN1 siRNA was transiently transfected into Eca-109 cells. The effects of transfection were detected by quantitative polymerase chain reaction and western blotting. The effects of STMN1 silencing by siRNA on the sensitivity of esophageal cancer cells Eca-109 to paclitaxel was tested by MTT and colony formation assays. Hoechst 33258 nuclear staining was used to investigate the differences in Eca109 cell apoptosis induced by paclitaxel. STMN1 siRNA was successfully transfected and the expression of STMN1 was inhibited. The sensitivity of STMN1 siRNA-transfected Eca-109 cells to paclitaxel was significantly increased $(P<0.01)$. The apoptosis of Eca-109 cells significantly increased following treatment with paclitaxel $(P<0.01)$. STMN1 silencing by siRNA may enhance the sensitivity of esophageal cancer cells Eca-109 to paclitaxel and induce apoptosis.
\end{abstract}

Key words: Chemosensitivity; Esophageal cancer cells; Paclitaxel; STMN1; Small interfering RNA 


\section{INTRODUCTION}

Esophageal squamous cell carcinoma is one of the most common cancers and is the world's sixth leading cause of death (Schweigert et al., 2013). China has one of the highest squamous cell carcinoma-related mortality rates, with esophageal squamous cell carcinoma ranking fourth in cancer-related deaths in China (Zhong et al., 2011). Studies have reported that patients with esophageal squamous cell carcinoma have poor prognosis for strict case-selection, neoadjuvant chemotherapy before surgery, and eradication therapy combined with surgery; the 5 -year survival rate is only approximately $20 \%$ (Crosby et al., 2009). Therefore, improving the effectiveness of chemoradiotherapy in esophageal squamous cell carcinoma and improving the survival and quality of life of patients through multi-modal treatment have become research hotpots. One of the main reasons for the failure of chemotherapy is the primary or secondary resistance of esophageal squamous cell carcinoma cells to chemotherapeutic drugs, but the mechanism is unclear. Therefore, further studies of the mechanisms of resistance in esophageal squamous cell carcinoma will help to improve clinical outcomes in patients with esophageal squamous cell carcinoma and provide an important theoretical basis for therapy and prognosis.

Paclitaxel is extracted from the bark of Taxus brevifolia; it can promote microtubule polymerization, inhibit microtubule degradation, arrest cell division cycle in the G2/M phase, and ultimately lead to tumor cell apoptosis; thus, it has a strong anti-tumor effect and has been used in a variety of cancer chemotherapy regimens, including esophageal squamous cell carcinoma (Yared et al., 2012). However, with increased clinical application, drug resistance to paclitaxel is also gradually increasing (Yared et al., 2012). Stathmin 1 (STMN1) is a microtubule destabilization phosphoprotein, which can alter microtubule dynamics during the mitotic phase and interphase (Rubin et al., 2004); its expression increases in many human cancer tissues, and it is involved in cell proliferation, cell motility, microtubule dynamic regulation, and cycle regulation (Rubin et al., 2004). STMN1 reportedly plays a key role in the resistance to paclitaxel in breast cancer and osteosarcoma (Alli et al., 2002; Ford et al., 2005; Wang et al., 2007; Carr et al., 2010), suggesting that STMN1 is also a drug resistance-related gene. Whether STMN1 promotes the resistance to paclitaxel in esophageal squamous cell cancer has not been thoroughly examined. In this study, small interfering RNA (siRNA) was used to silence STMN1 expression in esophageal squamous cell carcinoma Eca-109 cells. Drug susceptibility testing and apoptosis experiments were conducted to determine the sensitivity of esophageal squamous cell carcinoma cells to paclitaxel before and after intervention to preliminarily explore the effect of STMN1 silencing on the sensitivity of esophageal squamous cell carcinoma cells to paclitaxel.

\section{MATERIAL AND METHODS}

\section{Materials}

Human esophageal squamous cell carcinoma Eca-109 cells were purchased from Shanghai Cell Bank affiliated with the Chinese Science Institute (Shanghai, China). Trizol, Lipofectamine $^{\mathrm{TM}}$ 2000, and Opti-MEM $^{\circledR}$ I were purchased from Invitrogen (Carlsbad, CA, USA). RIPA lysis buffer, 5X protein sample buffer, sodium dodecyl sulfate-polyacrylamide gel electrophoresis preparation kit, phenylmethylsulfonyl fluoride, Ponceau stain, MTT, and crystal violet were purchased from Biyuntian Biotechnology Research Institute. The nitrocellulose 
membrane and electrochemiluminescence reagents were purchased from Millipore (Billerica, MA, USA). RPMI 1640 medium and fetal bovine serum were from Hyclone (Logan, UT, USA). Paclitaxel was purchased from Bristol-Myers Squibb (New York, NY, USA). The Hoechst staining kit was purchased from Biyuntian Biotechnology Research Institute. STMN1 siRNA and scramble siRNA were purchased from Shanghai GenePharma (Shanghai, China). The real-time quantitative reverse transcription-polymerase chain reaction (PCR) kit was purchased from Takara (Shiga, Japan). Anti-STMN1 antibody was purchased from Cell Signaling (Danvers, MA, USA). Anti- $\beta$ actin antibody was purchased from Santa Cruz Biotechnology (Santa Cruz, CA, USA). Horseradish peroxidase-labeled goat anti-rabbit and goat anti-mouse secondary antibodies were purchased from Zhongshan Golden Bridge Company (Beijing, China).

\section{Experimental methods}

\section{Cell culture}

Eca-109 cells were cultured in RPMI 1640 medium supplemented with $10 \%$ fetal calf serum. Except for cells that were not treated with antibiotics, 1 week before the transient transfection experiment, penicillin and streptomycin were added to the medium. Cells were cultured in an incubator at $37^{\circ} \mathrm{C}$ with $5 \% \mathrm{CO}_{2}$. All cells used in the experiment were in the logarithmic growth phase.

\section{Relevant primer sequences and quantitative PCR}

The Pubmed BLAST tool and relevant studies were used to design and synthesize primers: STMN1: sense: 5'-TAC ACT GCC TGT CGC TTG TC-3', antisense: 5'-GGG GAAAGG GGG AAT TCT GG-3'; glyceraldehyde 3-phosphate dehydrogenase (Ford et al., 2005): sense: 5'-CGG AGT CAA CGG ATT TGG TCG TAT-3', antisense: 5'-AGC CTT CTC CAT GGTGGT GAA GAC-3'. Trizol was used to extract RNA. The reaction system was prepared according to the specifications of the reverse transcription kit. The real-time quantitative PCR kit instructions were used to prepare the reaction systems and real-time quantitative PCR was subsequently performed.

\section{Relevant siRNA sequences and transfection experiments}

STMN1 siRNA was synthesized according to a previous study (Wang et al., 2007): sense: 5'-GAA ACG AGA GCA CGA GAA A-3', antisense: 5'-UUU CUC GUG CUC UCG UUU C-3', unrelated siRNA: sense: 5'-GCA AAA GAG CGA AAA G-3', antisense: 5'-CUU UUC GCU CUU UUG C-3'. Transient transfection steps were performed according Lipofectamine ${ }^{\mathrm{TM}} 2000$ instructions. We replaced the culture medium after $6 \mathrm{~h}$ transfection. After $72 \mathrm{~h}$, the cells were harvested for subsequent experiments.

\section{Western blot experiments}

The cells were trypsinized, collected, and lysed, and then total protein was extracted. We determined the protein concentration and performed sodium dodecyl sulfate-polyacrylamide gel electrophoresis, and transferred the proteins to the nitrocellulose membrane. Membranes 
were blocked in phosphate-buffered saline containing Tween-20 and 5\% skim milk. Samples were incubated with primary antibody at $-4^{\circ} \mathrm{C}$ overnight. The membrane was washed and the samples were incubated with secondary antibody at room temperature for $1 \mathrm{~h}$; after washing the membrane, electrochemiluminescence solution was added for observation.

\section{MTT drug susceptibility test}

The cells were digested with trypsin and collected. Next, the cells were seeded into 96well plates at a density of $5 \times 10^{3}$ cells/well, with $200 \mu \mathrm{L}$ medium in each well. After the cells had adhered for $16 \mathrm{~h}$, a concentration gradient of paclitaxel was added for in vitro drug sensitivity testing; parallel zero and control wells were included as controls. Plates were incubated for $72 \mathrm{~h}$; after $72 \mathrm{~h}$, MTT solution and dimethyl sulfoxide were added in order. The absorbance was detected using a microplate reader and analysis was performed.

\section{Plate colony formation assay}

The cells were digested with trypsin and collected. Next, the cells were seeded in 24-well plates at a density of $1 \times 10^{3}$ cells/well; after the cells had adhered for $16 \mathrm{~h}, 0.04 \mathrm{mM}$ paclitaxel was added for in vitro drug sensitivity testing. The medium was changed every 3 days and cells were incubated for a total of 14 days; 14 days later, medium was discarded and the cells were washed, fixed, and stained with $0.5 \%$ crystal violet; after photography with a digital camera, statistical analysis was conducted using Photoshop CS3 software.

\section{Hoechst 33258 nuclear staining experiments}

The cells were digested with trypsin and collected. Next, the cells were seeded in 6-well plates at a density of $1 \times 10^{6}$ cells/well; after the cells had adhered for $16 \mathrm{~h}, 0.01 \mathrm{mM}$ paclitaxel was added. The plates were placed in an incubator incubated for $36 \mathrm{~h}$ to induce apoptosis; $36 \mathrm{~h}$ later, according to the Hoechst staining kit instructions, staining and sheet sealing were performed. Photography was performed using a fluorescence microscope.

\section{Statistical methods}

SPSS 16.0 statistical software was used for experimental data analysis (SPSS, Inc., Chicago, IL, USA). The $t$-test was used for comparisons between groups. $\mathrm{P}<0.05$ was considered to indicate significant differences between groups. Experiments were repeated 3 times.

\section{RESULTS}

\section{Changes in expression levels of STMN1 in Eca-109 cells before and after transfection with STMN1 siRNA}

Quantitative PCR and western blotting test results showed that after $72 \mathrm{~h}$ STMN1 siRNA transfection, the expression level of STMN1 mRNA (Figure 1) and protein (Figure 2) significantly decreased in esophageal squamous cell carcinoma Eca-109 cells. 


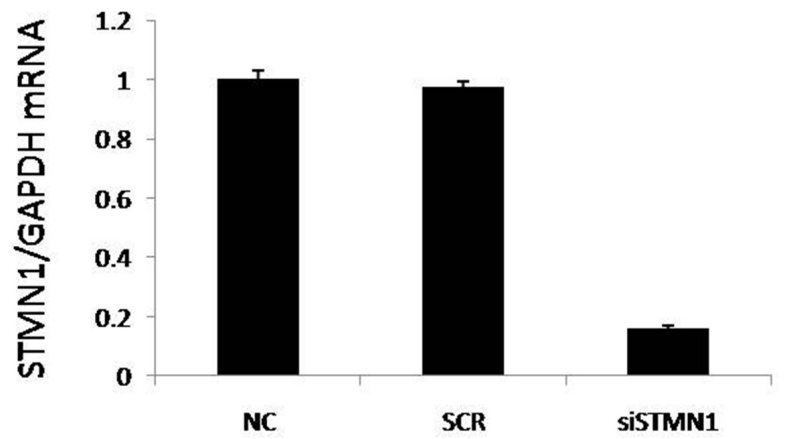

Figure 1. Changes of expression levels of mRNA in esophageal squamous cell carcinoma Eca-109 cells before and after transfection of STMN1. GAPDH was the internal control gene. NC: untransfected siRNA control group; SCR: unrelated control siRNA transfected group; siSTMN1: specific STMN1 siRNA transfection group.

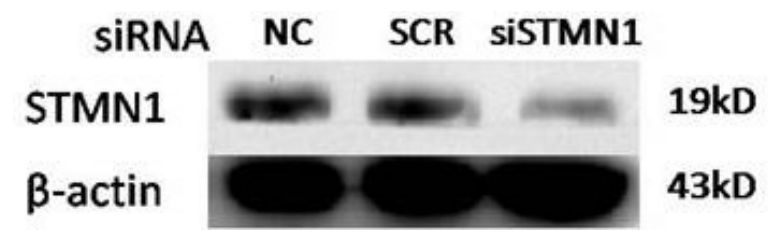

Figure 2. Changes of expression levels of STMN1 protein in esophageal squamous cell carcinoma Eca-109 cells before and after transfection of siRNA. $\beta$-actin was the internal control gene.

\section{MTT susceptibility results suggested that silencing of STMN1 increased the inhibition rate of paclitaxel on Eca-109 cells}

MTT drug susceptibility test results showed that after using specific siRNA to silence STMN1 expression, compared with the non-specific siRNA transfected group, the $50 \%$ inhibitory concentration of paclitaxel in esophageal squamous cell carcinoma Eca-109 cells decreased significantly $(P<0.01)$, indicating that the cells had increased in sensitivity to the drug (Figure 3 ).

\section{$I_{50(P<0.01)}$}

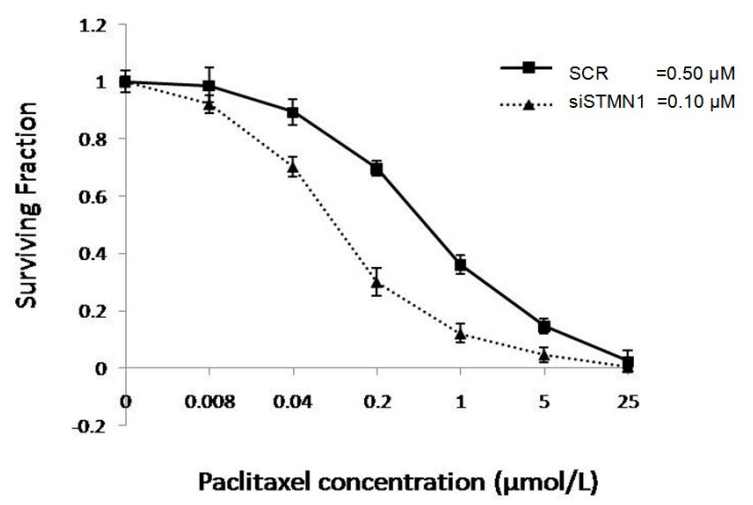

Figure 3. Eca-109 cell increased its sensitivity to paclitaxel after silencing STMN1. 


\section{Results of plate colony formation suggested that silencing STMN1 could increase} the sensitivity of Eca-109 cells to paclitaxel

The results of plate colony formation showed that after using specific siRNA to silence the expression of STMN1, compared with the nonspecific siRNA group, the number of plate colonized esophageal squamous cell carcinoma Eca-109 cells treated with $0.04 \mathrm{mM}$ paclitaxel was reduced significantly (Figure 4).
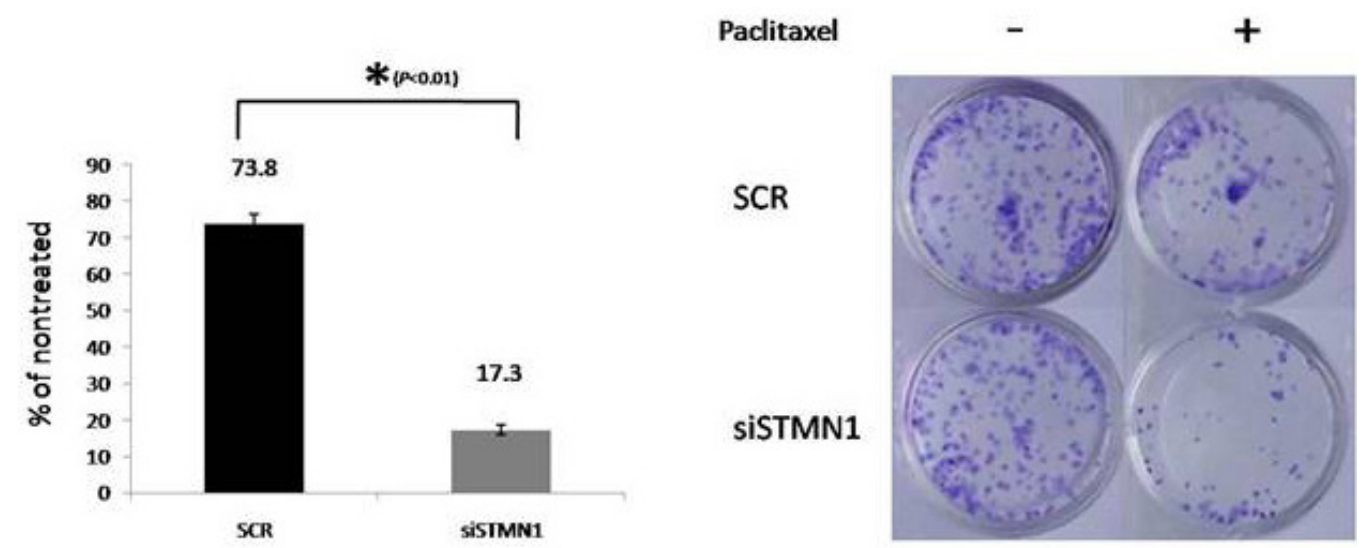

Figure 4. After silencing STMN1, the number of colony-formed esophageal squamous cell carcinoma Eca-109 cell reduced under the effect of paclitaxel. Left histogram was the statistical result; the right side were representative plate colony stained photos.

\section{After silencing of STMN1, apoptosis of Eca-109 cells increased in response to paclitaxel treatment}

Compared with the control group and unrelated siRNA transfection group, Eca-109 cells transfected with specific STMN1 siRNA and treated with $0.1 \mathrm{mM}$ paclitaxel for $36 \mathrm{~h}$ showed strong nuclear staining following Hoechst 33258 staining and the number of fragmented cells increased significantly (Figure 5), suggesting increased cell apoptosis.
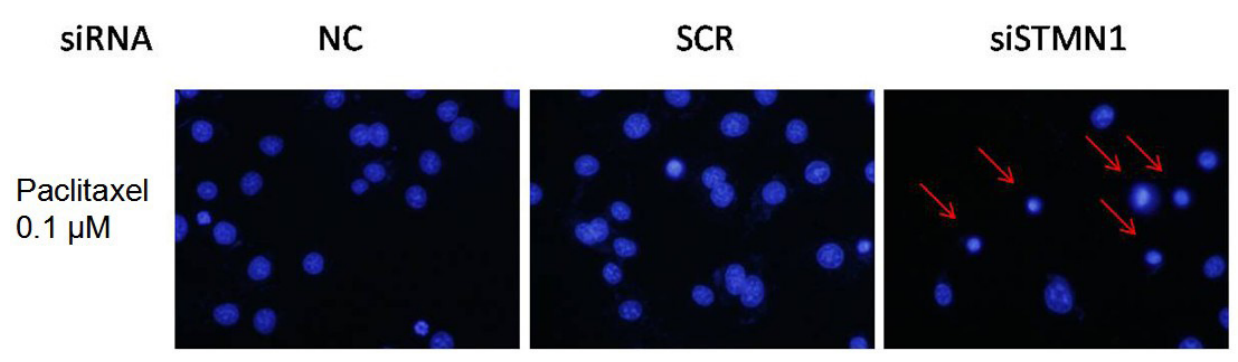

X400

Figure 5. After silencing STMN1, the apoptosis of esophageal squamous cell carcinoma Eca-109 cells induced by paclitaxel was increased. 


\section{DISCUSSION}

Paclitaxel is representative of taxanes and has been widely used in chemotherapy regimens for esophageal squamous cell cancer and other malignancies (Jordan et al., 2002; Yared et al., 2012). However, paclitaxel also has strong adverse reactions, such as allergic reactions, alopecia, bone marrow suppression, and peripheral neuropathy (Gligorov et al., 2004), limiting its application. In recent years, primary and secondary resistance of paclitaxel in cancer therapy has gradually attracted attention. Reducing the incidence of paclitaxel resistance but achieving the same killing effect on tumor cells at a lower concentration is important for the clinical treatment of esophageal squamous cell carcinoma.

STMN1, also known as stathmin 1, tumor protein 18, metablasin, p18, and p19, is encoded by the STMN1 gene located on human chromosome 1p36.1. STMN1 is a microtubule destabilization phosphoprotein affecting the microtubule dynamics during the mitotic phase and interphase (Rubin et al., 2004). STMN1 is involved in regulating mitotic microtubules in normal cell physiology; however, in tumor cells, STMN1 disorders often occur, resulting in dysfunction of the mitotic spindle and resistance to paclitaxel (McGrogan et al., 2008). In recent years, studies have reported that STMN1 promotes paclitaxel resistance in breast cancer and osteosarcoma (Alli et al., 2002; Ford et al., 2005; Wang et al., 2007; Carr et al., 2010). Therefore, we hypothesized that STMN1 is also involved in the resistance of esophageal squamous carcinoma cells to paclitaxel.

In this study, STMN1 siRNA was transiently transfected into esophageal squamous cell carcinoma Eca-109 cells using the liposome transfection method; quantitative PCR and western blotting revealed the effect of gene interference. After transfection, mRNA levels and protein levels off STMN1 decreased significantly. Further, drug sensitivity experiments showed that after silencing STMN1 expression, the chemosensitivity of esophageal squamous cell carcinoma Eca109 cells to paclitaxel significantly increased. Thus, by effectively silencing STMN1 expression, lower concentrations of paclitaxel may equally and effectively kill esophageal cancer cells, thus helping to reduce the clinical dosage of chemotherapy drugs, reduce the incidence of adverse reactions, and improve the therapeutic effectiveness. In the detection of apoptosis, we observed that after silencing STMN1 expression, the apoptosis of esophageal squamous cell carcinoma Eca-109 cells induced by paclitaxel was significantly increased, indicating that STMN1 mediates the resistance mechanism by reducing esophagus squamous cell apoptosis induced by paclitaxel.

In summary, STMN1 played a key role in the chemosensitivity of esophageal squamous cell carcinoma cells to paclitaxel; effective siRNA silencing of STMN1 may enhance the sensitivity of esophageal squamous cell carcinoma to paclitaxel and increase the apoptosis of esophageal squamous cell carcinoma cells in chemotherapy. The results of this study provide a further understanding of the mechanism of the resistance of esophageal squamous cell carcinoma to paclitaxel, and provides a theoretical basis for reducing paclitaxel adverse reactions and chemotherapy resistance in the clinic.

\section{Conflicts of interest}

The authors declare no conflict of interest.

\section{ACKNOWLEDGMENTS}

Research supported by the National Natural Science Foundation of China (\#81302067 
and \#81302164), the Natural Science Foundation of Guangdong Province (\#2014A030313595), the Science and Technology Plan Project of Guangdong Province (\#2013B021800049), and the Science and Technology Plan Project of Fujian Province (\#2012J05157).

\section{REFERENCES}

Alli E, Bash-Babula J, Yang JM and Hait WN (2002). Effect of stathmin on the sensitivity to antimicrotubule drugs in human breast cancer. Cancer Res. 62: 6864-6869.

Crosby T, Evans M, Gillies RS and Maynard ND (2009). The management of a patient with an operable carcinoma of the oesophagus. Ann. R. Coll. Surg. Engl. 91: 366-370.

Carr JR, Park HJ, Wang Z, Kiefer MM, et al. (2010). Foxm1 mediates resistance to herceptin and paclitaxel. Cancer Res. 70: 5054-5063.

Ford J, Jiang M and Milner J (2005). Cancer-specific functions of sirt1 enable human epithelial cancer cell growth and survival. Cancer Res. 65: 10457-10463.

Gligorov J and Lotz JP (2004). Preclinical pharmacology of the taxanes: Implications of the differences. Oncologist 2: 3-8.

Jordan MA (2002). Mechanism of action of antitumor drugs that interact with microtubules and tubulin. Curr. Med. Chem. Anticancer Agents 2: 1-17.

McGrogan BT, Gilmartin B, Carney DN and McCann A (2008). Taxanes, microtubules and chemoresistant breast cancer. Biochim. Biophys. Acta 1785: 96-132.

Rubin Cl and Atweh GF (2004). The role of stathmin in the regulation of the cell cycle. J. Cell. Biochem. 93: 242-250.

Schweigert M, Dubecz A and Stein HJ (2013). Oesophageal cancer--an overview. Nat. Rev. Gastroenterol. Hepatol. 10: $230-244$.

Wang R, Dong K, Lin F, Wang X, et al. (2007). Inhibiting proliferation and enhancing chemosensitivity to taxanes in osteosarcoma cells by rna interference-mediated downregulation of stathmin expression. Mol. Med. 13: 567-575.

Yared JA and Tkaczuk KH (2012). Update on taxane development: New analogs and new formulations. Drug Des. Devel. Ther. 6: 371-384.

Zhong X, Tan JJ and Xu ZX (2011). Esophageal squamous cell carcinoma etiology research progress of epidemiology. Quan Guo Zhong Liu Liu Xing Bing Xue He Zhong Liu Bing Yin Xue Xue Shu Hui Yi Lun Wen Ji. 22: 1-10-17. 